\title{
Food allergy and food-induced anaphylaxis in children: an increasing critical public health issue
}

Sooyoung Lee, MD, PhD

Department of Pediatrics, Ajou University School of Medicine, Suwon, Korea

Immunoglobulin E-mediated food allergies (FAs) including food-induced anaphylaxis are among the world's most serious health issues with life-threatening potential. ${ }^{1)}$ Why is FA an important social health problem? First, despite ongoing research into immunological therapeutic options, FA management is currently based on strict or proper avoidance of confirmed food allergens. Second, the appropriate response or treatment to accidental exposure to culprit foods/components is not always feasible. Furthermore, although fatalities from FA reactions in school are uncommon, school meals often induce allergic reactions in FA patients throughout the school setting, thus carrying definite risks for inducing anaphylaxis. This situation is more troublesome in nursery school and kindergarten because the prevalence of FA is much higher in infants and toddlers than in older children and adolescents. ${ }^{1,2)}$

FA prevalence and severity have notably increased during the past few decades, and the doctor-diagnosed FA prevalence was around 5\% in school children and adolescents in Korea. Furthermore, 25\% or more of FA patients experience anaphylaxis in childhood. ${ }^{1,2)}$ Anaphylaxis is a serious, rapid-onset systemic allergic reaction with fatal potential, and recent data suggest that its incidence related to food is increasing in many countries. In Korea, the prevalence of anaphylaxis has been increased and its awareness has been addressed among doctors, patients, parents, and society during the past 10 years. The prevalence of anaphylaxis in all ages was 20.55 in 2010, and 35.33 per 100,000 person-years in 2014. The overall prevalence increased 1.7-fold, with a higher rate of increase in children, particularly those aged 0-2 years. ${ }^{3)}$

According to a hospital-based multicenter study of anaphylaxis in Korean children (aged 0-18 years) between 2009 and 2013, 991 cases were reported, with 63.9\% involving patients younger than 6 years of age. ${ }^{4)}$ Similar to other countries, food was the most common anaphylaxis trigger (74.7\%) in Korean children. In another recent hospital-based multicenter study on FA in Korean children (aged 0-18 years, September 2014 to August 2015), the 3 most common causative foods differed among age groups. ${ }^{2)}$ In young children (0-2 years), cow's milk and hen's eggs accounted for more than two-thirds of FA cases; in preschool children 3-6 years of age, walnuts (15.0\%) were the most common cause of FA, followed by hen's eggs (13.5\%) and cow's milk (11.5\%); in children 7-12 years of age, walnuts and hen's eggs (10.4\% each) were the most common causes of FA, followed by cow's milk (9.0\%) and shrimp (8.2\%); and in adolescents 13-18 years of age, buckwheat (14.0\%) was the most common cause of FA, followed by crustaceans. The proportion of anaphylaxis varied from 15.4\% (soybean) to $67.7 \%$ (buckwheat). Regarding the acute onsite management of FA, the intramuscular injection of epinephrine is the first-line treatment for acute severe systemic reactions such as anaphylaxis. For children $\geq 15 \mathrm{~kg}$ who are diagnosed to food-induced anaphylaxis, selfinjectable/auto-injectable epinephrine prescriptions are strongly recommended to ensure the proper management of future occurrences. ${ }^{1,35}$

Despite growing awareness and the availability of general guidelines, the optimal elimination of causative food and control/prevention of FA reactions by meal services is not practically easy. ${ }^{1,5)}$ In the special aspect of school meal services, the government, and school requires
Corresponding author: Sooyoung Lee, MD, PhD Department of Pediatrics, Ajou University Hospital, Ajou University School of Medicine, 164 World cup-ro, Yeongtong-gu, Suwon 16499, Korea Tel: +82-31-219-5160

Fax: +82-31-219-5169

E-mail: jsjs87@ajou.ac.kr https://orcid.org/0000-0003-1734-4101

Received: 14 May, 2019

Revised: 6 November, 2019

Accepted: 12 November, 2019
Copyright $(\odot 2019$ by The Korean Pediatric Society

This is an open-access article distributed under the terms of the Creative Commons Attribution NonCommercial License (http://creativecommons.org/ licenses/by-nc/4.0/n which permits unrestricted noncommercial use, distribution, and reproduction in any medium, provided the original work is properly cited. 
policies and standardized protocols for the effective prevention and preparedness strategies for FA and food-induced anaphylaxis. In addition to readiness for action, education about FA reactions and pre-hospital acute management for patients is required for teachers and all people surrouding patients. ${ }^{1,5)}$ For example, teachers/friends are able to detect the anaphylactic reaction onsite and can help to administer the epinephrine auto-injector to the patient in the school setting. In Korea, the Allergenic Food Labeling Act contains 21 items in the commercial food marketing fields and school meal services under the control of the Korea Ministry of Food and Drug Safety and the Korea Ministry of Education. Furthermore, an epinephrine autoinjector (Jext) is imported and commercially available in Korea. Thus, the patient can buy a Jext according to one's body weight (Jext 0.15 for around 15-29 kg, Jext 0.3 for $>30 \mathrm{~kg}$ ) with a doctor's prescription (Korea Orphan Drug Center, http://www.kodc.or.kr). Recently, the revised "School Health Act, article 15" states that the school nurse can manage emergent conditions and provide life-saving medication such as insulin injections for hypoglycemia or epinephrine injections (auto-injector) for anaphylaxis. This law protects nurses from civil and criminal liability after providing these medical services.

Taken together, it is very encouraging that we have policies and guidance for preventing and managing FA and food-induced anaphylaxis in the school setting. However, effort is still required to ensure the effective implementation of all standards in the real world and educate patients, parents, teachers, school and medical per- sonnel, nutritionists, and food manufacturers. For this system to work properly, the pediatrician should be armed with knowledge and devotion.

\section{Conflicts of interest}

No potential conflict of interest relevant to this article was reported.

\section{References}

1. Lee S. Food allergy in Korean children: focus on IgE-mediated food allergy. J Korean Med Assoc 2017;60:242-8.

2. Jeong K, Kim J, Ahn K, Lee SY, Min TK, Pyun BY, et al. Age-based causes and clinical characteristics of immediate-type food allergy in Korean children. Allergy Asthma Immunol Res 2017;9:423-30.

3. Jeong K, Lee JD, Kang DR, Lee S. A population-based epidemiological study of anaphylaxis using national big data in Korea: trends in agespecific prevalence and epinephrine use in 2010-2014. Allergy Asthma Clin Immunol 2018;14:31.

4. Lee SY, Ahn K, Kim J, Jang GC, Min TK, Yang HJ, et al. A multicenter retrospective case study of anaphylaxis triggers by age in Korean children. Allergy Asthma Immunol Res 2016;8:535-40.

5. Mustafa SS, Russell AF, Kagan 0, Kao LM, Houdek DV, Smith BM, et al. Parent perspectives on school food allergy policy. BMC Pediatr 2018;18:164. 